\title{
Die Krux mit Zielkonflikten
}

\section{Für eine Revision des Integrativen Konzepts nachhaltiger Entwicklung}

\author{
Marc Dusseldorp, Karlsruher Institut für Technologie (KIT), Institut für Technikfolgenabschätzung und Systemanalyse (ITAS), \\ Karlstraße 11, 76133 Karlsruhe (marc.dusseldorp@kit.edu)
}

Das Integrative Konzept nachhaltiger Entwicklung zählt zu den prominentesten Ansätzen zur Operationalisierung des Nachhaltigkeitsleitbilds im deutschsprachigen Raum. Seit seiner Entwicklung um die Jahrtausendwende ist es in zahlreichen Projekten angewandt worden und hatte somit eine beträchtliche Reichweite. Eine kritische Analyse und Revision des Konzepts steht indes noch aus - was nicht zuletzt mit Blick auf die bestehenden Anwendungsprobleme Not täte. Hierzu möchte der vorliegende Artikel einen Beitrag leisten. Dabei zeigt sich, dass die Analyse des Integrativen Nachhaltigkeitskonzepts auch wichtige Impulse für die Nachhaltigkeitsdebatte im Allgemeinen und für den Umgang mit Zielkonflikten im Besonderen zu geben vermag. Insbesondere wird deutlich, dass das Nachhaltigkeitsleitbild mittels (nicht abwägbarer) Satisfizierungsnormen zu operationalisieren ist: Nur diese sind dazu geeignet, die Entwicklung hinreichend wirksamer Maßnahmen zur Realisierung einer nachhaltigen Entwicklung anzuleiten.

\section{Goal Conflicts - No Way Around Them}

The Case for a Revision of the Integrative Concept of Sustainable Development

The Integrative Concept of Sustainable Development is amongst the most prominent attempts in German-speaking countries to operationalise the notion of sustainability. Since its development around the turn of the millennium it has been applied in numerous projects with a considerable impact. However, a critical analysis and revision of the concept is still pending - but would be necessary, not least in view of the arising application problems. This paper aims at contributing to this concern. I appears that the analyses of the Integrative Concept can also give new impulses to the sustainability debate in general as well as to the question of dealing with goal conflicts in particular. Most notably, the concept of sustainable development needs to be operationalised using satisficing norms which cannot be weighed up: Only these norms can guide the development of measures effective enough the realise a sustainable development.

Keywords: sustainability assessment, goal conflicts, weighing goals, rules and principles, sustainability scenarios

This is an article distributed under the terms of the Creative Commons Attribution License CCBY 4.0 (https://creativecommons.org/licenses/by/4.0/)

https://doi.org/10.14512/tatup.27.2.61

Submitted: 09. 03.2018. Peer reviewed. Accepted: 07.05.2018

\section{Einführung}

Das Integrative Konzept nachhaltiger Entwicklung kann auf eine mehr als 15-jährige Anwendungsgeschichte zurückblicken. Um die Jahrtausendwende von der Helmholtz-Gemeinschaft Deutscher Forschungszentren (HGF) entwickelt, hat es weit über deren Grenzen hinaus die Befassung mit dem Nachhaltigkeitsleitbild in Forschung und Lehre geprägt (Kopfmüller 2006). Seine beträchtliche Reichweite verdankt es nicht zuletzt groß angelegten Verbundprojekten mit Bezug zur Politikberatung (etwa dem HGF-Projekt „EnergyTrans“ zur deutschen Energiewende, Rösch et al. 2016), in denen das Konzept als normative Grundlage diente und entsprechenden Niederschlag in den Projektergebnissen fand.

Ungeachtet seiner praktischen Bedeutung ist das Integrative Nachhaltigkeitskonzept seit Erscheinen seiner konzeptionellen Hauptveröffentlichung (Kopfmüller et al. 2001) kaum kritisch analysiert worden - ein Workshop aus dem Jahr 2004 und der zugehörige Sammelband (Kopfmüller 2006) bilden hier die Ausnahme. Auch eine Überarbeitung hat bislang nicht stattgefunden. Allerdings war bereits in der Hauptveröffentlichung die Absicht formuliert worden, aus den Anwendungserfahrungen zu lernen und das Konzept auf dieser Basis weiterzuentwickeln. Dies gilt insbesondere für den Umgang mit Zielkonflikten: Die konzepteigene Abwägungsmethodik, so die Autoren bereits damals, stelle kein ,,ausgereiftes Abwägungsmodell“ dar, sondern lasse es lediglich ,,auf einer ziemlich abstrakten Ebene möglich erscheinen [...], Abwägungsprozesse nicht allein intuitiven Bewertungen anheim zu stellen, sondern begrifflich so präzise wie möglich zu fassen." Daher könne es bei der Anwendung des Konzepts ,nur darum gehen, mittels der vorgeschlagenen Methodik Erfahrungen zu sammeln und darauf Lernprozesse zu gründen“ (Kopfmüller et al. 2001, S. 180).

Vor diesem Hintergrund soll der vorliegende Artikel einen Beitrag zur Kritik des Integrativen Nachhaltigkeitskonzepts leisten sowie Anstöße zu dessen Überarbeitung geben. ${ }^{1}$ Dabei werden inhaltliche Fragen, etwa welche Belange - Chancengleich-

1 Es handelt sich dabei um einen Auszug aus der Dissertation „Zielkonflikte der Nachhaltigkeit. Zur Methodologie wissenschaftlicher Nachhaltigkeitsbewertungen“" (Dusseldorp 2017). Für eine gründlichere Analyse des Integrativen Nachhaltigkeitskonzepts wie auch der Zielkonfliktproblematik sei auf diese Arbeit verwiesen. 
heit, Landschaftsschutz u. a. - überhaupt als nachhaltigkeitsrelevant gelten dürfen, weitgehend außer Acht gelassen. ${ }^{2}$ Stattdessen soll die (normtheoretische) Frage nach dem Status oder „Charakter" der Nachhaltigkeitsnormen im Mittelpunkt stehen. Diese Frage spielt, trotz ihrer entscheidenden Rolle für das Problem des Umgangs mit Zielkonflikten, in konzeptionellen Beiträgen zur Nachhaltigkeitsdebatte bislang eine untergeordnete Rolle. Sie ins Zentrum zu rücken ist - so wird sich zeigen - für einen wohlbegründeten Umgang mit Zielkonflikten sowie letztlich generell für angemessene Nachhaltigkeitsbewertungen unerlässlich.

\section{Nachhaltigkeit: Mindestbedingungen menschenwürdigen Lebens}

Der Aufbau des Integrativen Nachhaltigkeitskonzepts ist in den vergangenen Jahren in einer Vielzahl von Publikationen dargestellt worden. Auf diese sei für detailliertere Ausführungen verwiesen (ausführlich Kopfmüller et al. 2001, kompakt z. B. Coenen und Grunwald 2003). An dieser Stelle soll ein Blick auf einige zentrale Charakteristika genügen:

Der namensgebende ,integrative“ Zugang besteht im HGF-Konzept darin, das Nachhaltigkeitsleitbild nicht wie üblich für drei oder mehr Dimensionen separat zu operationalisieren, sondern Dimensionen übergreifende konstitutive Elemente nachhaltiger Entwicklung - inter- und intragenerative Gerechtigkeit unter den Randbedingungen Globalität und Anthropozentrik - an den Beginn der Operationalisierung zu stellen. Diese konstitutiven Elemente werden sodann in Anlehnung
Ein zweites, wichtiges Charakteristikum des Integrativen Nachhaltigkeitskonzepts ist sein ,minimalistisches“ Nachhaltigkeitsverständnis: „Nachhaltigkeit besteht substanziell in der dauerhaften Sicherung von Mindestbedingungen menschenwürdigen Lebens. Diese Mindestbedingungen lassen sich unter drei ,generelle Ziele" der Nachhaltigkeit subsumieren und als substanzielle Nachhaltigkeitsregeln formulieren [...]“ (Kopfmüller et al. 2001, S. 118). Damit will das Konzept Ansätzen entgegentreten, die den Begriff einer nachhaltigen Entwicklung inhaltlich überfrachten und so nicht nur die Stoßrichtung des Leitbilds verfehlen, sondern auch dessen praktische Wirksamkeit beeinträchtigen. Es soll stattdessen ,lediglich einen ,Wohlfahrtssockel““ garantieren, auch wenn es ,noch andere legitime und erstrebenswerte Ziele gibt, deren Erfüllung nicht als konstitutiv für das Leitbild der Nachhaltigkeit angesehen wird" (Kopfmüller et al. 2001, S. 177). Freilich bezieht sich die Forderung des Integrativen Konzepts auf alle Menschen: Mindestbedingungen menschenwürdigen Lebens sind für die heute lebende Generation ebenso herzustellen wie für künftige Generationen zu bewahren. Darüber hinaus gelten dem Konzept die 15 substanziellen Nachhaltigkeitsregeln als notwendig und hinreichend für das Vorliegen einer nachhaltigen Entwicklung: „,[W]enn alle Regeln erfüllt sind, ist das Postulat der nachhaltigen Entwicklung realisiert, bei Verletzung bereits einer Regel nicht" (Coenen und Grunwald 2003, S. 21).

Die skizzierte Operationalisierung soll dazu dienen, ,nachhaltige Entwicklung als einen Begriff mit Unterscheidungskraft zu bestimmen“ (Coenen und Grunwald 2003, S. 55) und damit der verbreiteten Kritik zu begegnen, der Gebrauch des Begriffs sei zu unscharf, ja nahezu beliebig. Unterschieden werden

\section{Dem Integrativen Nachhaltigkeitskonzept zufolge besteht Nachhaltigkeit substanziell in der dauerhaften Sicherung von Mindestbedingungen menschenwürdigen Lebens.}

an Brown-Weiss' Theorie intergenerativer Gerechtigkeit (1989) in drei generelle Ziele nachhaltiger Entwicklung übersetzt, nämlich Sicherung der menschlichen Existenz, Erhaltung des gesellschaftlichen Produktivpotenzials sowie Bewahrung der Entwicklungs- und Handlungsmöglichkeiten. Der normative Kern des Integrativen Konzepts, ein Satz von 15 substanziellen Nachhaltigkeitsregeln, ist schließlich als Präzisierung dieser generellen Ziele zu verstehen. Rund ein Viertel des Grundlagenbandes ist ausführlichen Erläuterungen zum Inhalt der 15 Nachhaltigkeitsregeln gewidmet.

2 Wenngleich das Konzept auch von einem kritischen Blick auf seinen Inhalt profitieren könnte. Die Forderung etwa, allen Mitgliedern einer Gesellschaft sei im Sinne des Nachhaltigkeitsleitbilds die Möglichkeit selbständiger Existenzsicherung zu gewähren, erscheint vor dem Hintergrund der Debatten um ein bedingungsloses Grundeinkommen durchaus fragwürdig. soll konkret zwischen nachhaltigen und nicht nachhaltigen $\mathrm{Zu}$ ständen, wobei das Attribut „,nachhaltig“ „,ohne weitere Erläuterung nur dem gesellschaftlichen (globalen) Entwicklungspfad im Ganzen zugeschrieben werden“ könne (Coenen und Grunwald 2003, S. 64). Des Weiteren sollen politische Maßnahmen, Technologien sowie andere Bewertungsgegenstände mit Handlungscharakter - im Folgenden generell „Maßnahmen“ genannt dahingehend beurteilt werden können, ob ihre Realisierung im Lichte des Nachhaltigkeitsleitbildes empfehlenswert erscheint oder nicht. Ist dies der Fall, so kann ihnen im Sinne des Integrativen Konzepts ein „Beitrag“ zu einer nachhaltigen Entwicklung attestiert werden. ${ }^{3}$

3 Daneben sieht das Integrative Nachhaltigkeitskonzept auch komparative Bewertungen (sowohl der gesellschaftlichen Entwicklung als auch von Maß- - 
Für die Anwendung des Konzepts ist die oben genannte Charakterisierung der substanziellen Regeln als notwendige und hinreichende Bedingungen für nachhaltige Entwicklung entscheidend - zusammen mit der Frage der Abwägbarkeit der Nachhaltigkeitsregeln, auf die unten näher eingegangen wird. Ohne eine solche Charakterisierung wäre lediglich gesagt, dass nachhaltige Entwicklung etwas mit den Belangen Ressourcenschutz, Chancengleichheit usw. „zu tun“ hat und dass diese Belange bei nachhaltigkeitsorientierten Entscheidungen ,zu berücksichtigen" sind. Die gewünschte Unterscheidungskraft ließe sich so nicht schaffen. Die substanziellen Regeln, die Mindestbedingungen menschenwürdigen Lebens formulieren, müssen jedoch alle erfüllt sein, damit der gesellschaftliche Entwicklungspfad im Ganzen als „nachhaltig“ gelten kann. Dies ist die Richtschnur für Nachhaltigkeitsbewertungen mit dem Integrativen Konzept.

\section{Anwendung ohne Abwägung: Das Integrative Konzept in der Praxis}

Es existiert eine breite Palette abgeschlossener Projekte, in denen das Integrative Nachhaltigkeitskonzept bereits angewandt wurde. Daher ist es nunmehr möglich, ein empirisch belastbares Bild der Probleme zu zeichnen, die bei seiner Anwendung auftreten. $^{4}$

Zunächst zu den Fällen, in denen die gesellschaftliche Entwicklung im Ganzen in den Blick genommen wurde, derjenige Untersuchungsgegenstand also, dem die Eigenschaft, nachhaltig zu sein, eigentlich zukommen kann. Dass eine nachhaltige Entwicklung derzeit nicht gegeben ist, darf als trivialer Befund gelten. Auch methodisch ist dieser Befund trivial, gilt es doch lediglich zu prüfen, ob sämtliche substanziellen Nachhaltigkeitsregeln erfüllt sind. Von weit größerer Praxisrelevanz ist, dass im Zuge solcher Untersuchungen auch Nachhaltigkeitsdefizite identifiziert werden (für Deutschland in Coenen und Grunwald 2003). Welche Regeln sind in welchem Maße beeinträchtigt? Dies zu wissen, ist für die Entwicklung einer nachhaltigkeitsorientierten Politik gewiss eine wichtige Grundlage. Zielkonflikte werden bei den vorliegenden Bewertungen der gesellschaftlichen Entwicklung indes nicht zum Thema (was letztlich nicht verwundern kann, s. Abschnitt ,Zwei Typen von Normen“) - ein Abwägungsproblem stellt sich hierbei also nicht.

Anders verhält es sich, wenn das Integrative Nachhaltigkeitskonzept für die Bewertung von Maßnahmen angewandt wird. Hier spielen Zielkonflikte eine zentrale Rolle - allerdings in einem gänzlich verschiedenen Sinn: Während bei der Bewertung der gesellschaftlichen Entwicklung solche Fälle als „Zielkonflikte" bezeichnet werden, in denen die gleichzeitige Erfüllung

> nahmen) vor. Diese sind methodologisch jedoch gegenüber den o.g. klassifikatorischen Bewertungen nachrangig, weshalb im Folgenden nur auf letztere eingegangen wird.

4 Dem vorliegenden Artikel liegt die Auswertung von Dusseldorp (2017,

S.137-140) zugrunde, die zumindest den größten Teil der Anwendungen des Integrativen Konzepts erfasst haben dürfte. aller Nachhaltigkeitsregeln unmöglich erscheint, ist bei Maßnahmenbewertungen von ,Zielkonflikten“ die Rede, wenn von einer Maßnahme divergierende, d.h. teils positive, teils negative Wirkungen auf die inhaltliche Realisierung der Nachhaltigkeitsregeln ausgehen. Zielkonflikte dieses Typs wurden bei Anwendungen des Integrativen Nachhaltigkeitskonzepts zahlreich identifiziert.

Die bloße Identifikation ist indes nicht der Beginn eines weitergehenden Umgangs mit Zielkonflikten in der Anwendung des Integrativen Konzepts, sondern bereits der Endpunkt. Das konzepteigene Abwägungsverfahren wird in den Projekten nicht (auch nur versuchsweise) angewandt. Es wird weder darauf Bezug genommen, noch wird das Ausbleiben von Abwägungen selbst Gegenstand einer Reflexion. ${ }^{5}$ Stattdessen wird in Zielkonflikt-Fällen allenfalls die Empfehlung abgegeben, ,Win-win-Lösungen“" zu entwickeln, d. h. Maßnahmen, deren Auswirkungen auf die Realisierung der Nachhaltigkeitsregeln ausschließlich positiv sind. Allerdings ist die generelle Empfehlung von Win-win-Maßnahmen weder methodisch haltbar (Dusseldorp 2017, S. 148-149), noch gibt sie eine Antwort auf die Frage, ob die ursprünglich zu bewertende Maßnahme insgesamt positiv oder negativ zu bewerten ist, mit anderen Worten: ob ihre Realisierung im Lichte des Nachhaltigkeitsleitbilds „unterm Strich“ empfehlenswert erscheint oder nicht.

Solche Gesamtbewertungen von Maßnahmen stellen jedoch einen der wichtigsten Zwecke von Nachhaltigkeitskonzepten dar. Nachhaltigkeitsdefizite zu kennen ist Grundlage für nachhaltigkeitsorientiertes Handeln - aber nicht schon für sich genommen einer nachhaltigen Entwicklung zuträglich, sondern erst dann, wenn geeignete Maßnahmen folgen. Freilich kann sich die Anwendung des Integrativen Nachhaltigkeitskonzepts auf die Identifikation von Zielkonflikten beschränken und die Bildung von $\mathrm{Ge}$ samturteilen dem Rezipienten der Studienergebnisse überlassen. Es ist jedoch der explizite Anspruch des Konzepts, Maßnahmen insgesamt zu bewerten, und dies auch aus gutem Grund: Wenn die wissenschaftliche Befassung mit dem Nachhaltigkeitsleitbild Empfehlungen für dessen angemessene Interpretation sowie für nachhaltigkeitsorientiertes Handeln erarbeiten soll, kann sie nicht vor dem methodisch anspruchsvollsten und zugleich praktisch bedeutsamsten Anwendungsproblem kapitulieren.

\section{Zwei Typen von Normen: Regeln und Prinzipien}

Vor diesem Hintergrund liegt die Frage auf der Hand, ob die Anwendungsprobleme des Integrativen Konzepts auf den unausgereiften Stand seines Abwägungsverfahrens zurückzuführen sind. $\mathrm{Zu}$ Beantwortung dieser Frage ist zunächst ein genauerer Blick auf den theoretischen Hintergrund des Verfahren zu werfen.

5 Eine Ausnahme bildet allein Parodi (2008), der angesichts der Unzulänglichkeiten des vorliegenden Abwägungsverfahrens ein eigenes Verfahren skizziert ohne dieses jedoch auf die in der eigenen Arbeit identifizierten Zielkonflikte anzuwenden. 
Das Abwägungsverfahren des Integrativen Konzepts basiert auf den - in der Rechtswissenschaft prominenten - grundrechtstheoretischen Arbeiten Robert Alexys (1996) sowie auf der Gerechtigkeitstheorie von dessen Schüler Nils Jansen (1998). Für beide Theorien ist die Unterscheidung zweier Typen von Normen zentral: Regeln und Prinzipien. Prinzipien stellen Optimierungsgebote dar, d. h. Normen, ,die gebieten, daß etwas in einem relativ auf die rechtlichen und tatsächlichen Möglichkeiten möglichst hohen Maße realisiert wird“ (Alexy 1996, S. 75). Was dies bedeutet, wird in Kollisionsfällen besonders deutlich: Zwei Prinzipien führen dann, je für sich genommen, zu einander widersprechenden Ergebnissen. Tierversuche etwa sind er- sich, dass Alexys ansonsten sehr präzise Terminologie eine Schwachstelle besitzt. Diese tritt besonders deutlich zu Tage, wenn Alexy Regeln als Normen charakterisiert, „die stets nur entweder erfüllt oder nicht erfüllt werden können“, Prinzipien hingegen als Normen, die ,,in unterschiedlichen Graden erfüllt werden können“ (Alexy 1996, S. 76). Beide Charakterisierungen reflektieren nicht die Doppeldeutigkeit des Ausdrucks „Erfüllung": Normen sind erfüllt, wenn sie nicht verletzt bzw. nicht befolgt sind; und sie besitzen einen Inhalt, der (bisweilen in unterschiedlichen Graden) erfüllt sein kann. Um Missverständnisse zu vermeiden, sollte daher „Erfüllung“ für den Gegenbegriff zu „Verletzung“ reserviert sein und in Bezug auf den Normin-

\section{Die Unterscheidung von Regeln und Prinzipien ist zentral}

\section{für das Abwägungsverfahren des Integrativen Nachhaltigkeitskonzepts - wie auch generell für den Umgang mit Zielkonflikten.}

laubt, wenn das Prinzip der Forschungsfreiheit für sich genommen wird, nach dem Prinzip des Tierschutzes hingegen verboten. Jedes Prinzip begrenzt die rechtliche Möglichkeit der Erfüllung des anderen. $\mathrm{Ob}$ das betreffende Handeln im Falle einer Prinzipienkollision erlaubt oder verboten ist, muss mittels einer Abwägung geklärt werden. Prinzipien sind also abwägbare Normen. Selbst weitreichende inhaltliche Beeinträchtigungen durch gegenläufige Normen können bei Prinzipien gerechtfertigt sein. Die Gültigkeit oder Geltung der Prinzipien ist von Abwägungen indes nicht berührt (Kollisionsfälle setzen die Geltung der kollidierenden Prinzipien voraus): Die Abwägung legt lediglich fest, welchem Prinzip im konkreten Fall der Vorrang gebührt.

Dem gegenüber handelt es sich bei Regeln um Normen, die gebieten, ,genau das zu tun, was sie verlang[en], nicht mehr und nicht weniger" (Alexy 1996, S. 76). Regeln können somit als Satisfizierungsgebote (March 1994) charakterisiert werden ein Begriff, den zwar Alexy nicht gebraucht, der jedoch Alexys Verständnis dieses Typs von Normen besonders treffend benennt: Einer Regel wird genügt, wenn „etwas“ - ihr Inhalt nicht im höchstmöglichen, sondern in einem bestimmten Maße realisiert wird. Ein Mehr an inhaltlicher Realisierung ist für ihre Erfüllung nicht erheblich, ein Weniger bedeutet zwingend ihre Verletzung. Regeln, deren Inhalt nicht graduell realisierbar ist (etwa Verkehrsregeln wie „Überhole stets links!“), sind gleichsam Grenzfälle dieser Definition. Konfligieren Regeln miteinander, so muss eine Ausnahmeklausel in eine der Regeln eingefügt oder aber mindestens eine der Regeln für ungültig erklärt werden. Abwägungen sind bei Regelkonflikten nicht möglich: Wird den Forderungen einer Regel nicht (in hinreichendem Maße) Genüge getan, so ist sie verletzt. Inhaltliche Beeinträchtigungen von Regeln können, anders als bei Prinzipien, nicht durch gegenläufige Normen gerechtfertigt werden.

Bei den Kurzbeschreibungen von Prinzipien und Regeln zeigt halt - das „etwas“, das im höchstmöglichen bzw. in einem bestimmten Maße zu realisieren ist - konsequent von „Realisierung“ gesprochen werden. Eine Charakterisierung, die dies berücksichtigt, könnte also lauten: Regeln sind Normen, die ein bestimmtes, absolutes Maß ihrer inhaltlichen Realisierung verlangen (und die dem entsprechend im Konfliktfall nicht abgewogen werden können). Prinzipien hingegen sind Normen, die ein relatives Maß ihrer inhaltlichen Realisierung - nämlich das höchstmögliche - fordern (und für die im Konfliktfall eine Abwägung zulässig ist).

\section{Sind die substanziellen Nachhaltigkeitsregeln wirklich (abwägbare) Prinzipien?}

Zurück zum Integrativen Konzept: Dort werden die substanziellen Nachhaltigkeitsregeln explizit als Prinzipien im Sinne Alexys charakterisiert. ${ }^{6}$ Es handle sich ,um Prinzipien, da sie (1) in Abhängigkeit von den tatsächlichen und rechtlichen Möglichkeiten in unterschiedlichem Maße erfüllt werden können und (2) im Konfliktfall keine der betroffenen Regeln für ungültig erklärt werden kann, sondern eine Güterabwägung erfolgen muss“ (Kopfmüller et al. 2001, S. 175). Inhaltliche Beeinträchtigungen einzelner Regeln könnten demnach durch gegenläufige substanzieller Nachhaltigkeitsregeln gerechtfertigt sein, was im Zuge von Abwägungen zu klären ist.

Was aber soll es bedeuten, dass die Nachhaltigkeitsregeln (verstanden als Prinzipien) ,,in unterschiedlichem Maße erfüllt

6 Dass sie gleichwohl „substanzielle Nachhaltigkeitsregeln“ genannt werden, wird mit der terminologischen Anschlussfähigkeit an die im Nachhaltigkeitsdiskurs verbreitete Rede von „ökologischen Managementregeln“ begründet. 
werden können“? „Erfüllung“ als Gegenbegriff zu „Verletzung“ ist begrifflich unmöglich graduell: Eine Norm ist erfüllt oder verletzt - ein Mehr oder Weniger ergibt hier keinen Sinn. Graduell erfüllbar (realisierbar) ist freilich der Inhalt zahlreicher Normen, so auch der substanziellen Nachhaltigkeitsregeln. Macht sie dies zu Prinzipien? Nein - dafür ist entscheidend, ob die Nachhaltigkeitsregeln ihre höchstmögliche inhaltliche Realisierung oder aber ihre inhaltliche Realisierung in einem bestimmten Maße verlangen. Hierzu macht die zitierte Textstelle jedoch keine Aussage.

Auch die darin angesprochenen Konfliktfälle, in denen „eine Güterabwägung erfolgen" müsse, werfen Fragen auf: Sind damit Fälle gemeint, in denen die gleichzeitige Erfüllung der substanziellen Nachhaltigkeitsregeln unmöglich erscheint? Falls ja: Wie könnten solche Konfliktfälle identifiziert werden? Dies ist schwer vorstellbar - denn wenn Nachhaltigkeit als etwas konzipiert wird, dessen Realisierung für möglich gehalten wird, muss von der gleichzeitigen Erfüllbarkeit der Nachhaltigkeitsregeln ausgegangen werden. Diese Annahme liegt dem Integrativen Konzept denn auch zugrunde; und sie kann generell durch Narrationen, die mögliche Wege hin zu einer nachhaltigen Entwicklung zeichnen (,Nachhaltigkeitsszenarien“), plausibilisiert werden (Dusseldorp 2017, S. 180-183, 204-210). Die bloß faktische Verletzung der Nachhaltigkeitsregeln ist nicht mit unmöglicher Erfüllbarkeit zu verwechseln. Gewiss sind die Regeln derzeit verletzt; dies allein ist jedoch kein Grund, an der Möglichkeit einer nachhaltigen Entwicklung zu zweifeln, sondern geradezu umgekehrt der Anlass, sich für deren Verwirklichung zu engagieren. Der oben angesprochene Befund, dass bei der Anwendung des Integrativen Konzepts zur Bewertung der gesellschaftlichen Entwicklung keine Zielkonflikte identifiziert wurden, wird vor diesem Hintergrund verständlich.

Häufig treten hingegen Zielkonflikte in dem zweiten, oben skizzierten Sinn auf: als Fälle, in denen Maßnahmen mit divergierenden Wirkungen auf die Realisierung der Nachhaltigkeitsregeln (verstanden als Prinzipien) einhergehen. Sind in der zitierten Textstelle diese „Konfliktfälle“ gemeint? Dann wäre nicht ersichtlich, weshalb im Anschluss eine „Güterabwägung“ entsprechend Alexys Abwägungsverständnis erfolgen müsste. Wenn Maßnahmen mit divergierenden Wirkungen einhergehen, so heißt dies nicht, dass die davon betroffenen Nachhaltigkeitsregeln nicht erfüllt werden - die Erfüllung hängt davon $\mathrm{ab}$, welche Maßnahmen darüber hinaus durchgeführt werden. In den meisten Fällen besteht eine große Kluft zwischen der Wirkmächtigkeit einzelner Maßnahmen und dem Anspruchsniveau der substanziellen Nachhaltigkeitsregeln. Das Ziel der $\mathrm{CO}_{2}$-Emissionsreduktion (als Teil der Regel zur nachhaltigen Nutzung der Umwelt als Senke) etwa lässt sich nur durch ein immenses Bündel von Maßnahmen - im In- und Ausland, im Strom-, Wärme- und Kraftstoffbereich, in Industrie und Privathaushalten - erreichen. Das Integrative Nachhaltigkeitskonzept verlangt lediglich, dass die substanziellen Regeln erfüllt werden. Daraus kann nicht ohne weiteres geschlossen werden, welchen Beitrag eine bestimmte Maßnahme dazu zu leisten hat.
Angesichts dessen wird deutlich, dass die Übertragung von Alexys Prinzipientheorie der Grundrechte auf das Abwägungsproblem im Integrativen Nachhaltigkeitskonzept auf einem Missverständnis basiert: Während im Grundrechtskontext tatsächlich Normen kollidieren, wird im Integrativen Konzept davon ausgegangen, dass die Nachhaltigkeitsnormen zugleich vollständig inhaltlich realisierbar sind - und mithin keine Kollisionen auftreten. Das Zielkonfliktproblem besteht hier vielmehr aus Fällen, in denen Maßnahmen mit divergierenden Wirkungen (auf die Realisierung der Nachhaltigkeitsregeln) einhergehen. Es ist somit auf einer gänzlich anderen Ebene zu verorten. ${ }^{7}$ Darüber hinaus sind grundlegende Zweifel an der Charakterisierung der Nachhaltigkeitsregeln als Prinzipien angebracht, denn die zitierte Begründung erwies sich als nicht schlüssig. Schließlich fragt sich auch: Weshalb sollten die Nachhaltigkeitsregeln abwägungsfähig sein, wenn von ihrer Realisierbarkeit ausgegangen wird?

\section{Regeln zur Operationalisierung von Nachhaltigkeit!}

Nun werden die Nachhaltigkeitsregeln im Integrativen Konzept zwar explizit als Prinzipien charakterisiert. Neben dieser abstrakten Bestimmung finden sich jedoch noch weitere, konkrete Charakterisierungen der Nachhaltigkeitsregeln.

Ein Beispiel zeigt sich in folgender Textstelle: ,[Es] liegt der vorliegenden Konzeption die Annahme zugrunde, dass die Regeln, da sie nur Mindestbedingungen der Nachhaltigkeit formulieren, stets zugleich erfüllbar seien“ (Kopfmüller et al. 2001, S. 179). Geht man davon aus, dass die Nachhaltigkeitsregeln tatsächlich Prinzipiencharakter haben, greift diese Begründung ins Leere: Ein System von Prinzipien ist, seine Konsistenz vorausgesetzt, immer erfüllbar; jedenfalls ist seine Erfüllbarkeit nicht davon abhängig, wie anspruchsvoll die Forderungen der einzelnen Prinzipien sind, d. h. ob diese „nur Mindestbedingungen der Nachhaltigkeit" fordern oder deutlich mehr. Geht man jedoch davon aus, dass die Nachhaltigkeitsregeln nicht Prinzipien, sondern Regeln (Satisfizierungsnormen) darstellen, ist die $\mathrm{Ar}$ gumentation schlüssig: Für die gleichzeitige Erfüllbarkeit von Normen, die in einem bestimmten Maße inhaltlich zu realisieren sind, ist es durchaus von Belang, wie anspruchsvoll die Forderungen sind.

7 Der Methodologie des Integrativen Nachhaltigkeitskonzepts mangelt es letztlich an einer konsequenten Unterscheidung seiner beiden Untersuchungsgegenstände: Bewertungen der gesellschaftlichen Entwicklung stellen auf die inhaltliche Realisierung der Nachhaltigkeitsregeln ab, Bewertungen von Maßnahmen hingegen auf deren Wirkung auf erstere. Entsprechend verhält es sich mit den beiden Zielkonfliktbegriffen, die im Integrativen Konzept kursieren, jedoch nicht in ihren Unterschieden reflektiert werden. Darüber hinaus lassen sich im Anschluss an o.g. Unterscheidung noch weitere terminologische Ungereimtheiten klären, etwa die doppeldeutige Verwendung der Rede von „Beiträgen zu einer nachhaltigen Entwicklung“, die zum einen auf gesellschaftliche Teilbereiche und Regionen (im Sinne eines Anteils am Ganzen), zum anderen auf Maßnahmen (im Sinne einer Wirkung) bezogen wird. 
Ein weiterer Hinweis liegt im Begriff des Nachhaltigkeitsdefizits. Wären die substanziellen Nachhaltigkeitsregeln tatsächlich Prinzipien, könnten Nachhaltigkeitsdefizite nicht einfach an ihrem Realisierungsgrad abgelesen werden: Beeinträchtigungen könnten ja durch gegenläufige Nachhaltigkeitsregeln gerechtfertigt sein. Um dies zu klären, wären Abwägungen erforderlich - die jedoch bei der Anwendung des Integrativen Nachhaltigkeitskonzepts nicht stattfinden. Stattdessen werden Defizite identifiziert, indem der Realisierungsgrad jeder Regel für sich genommen betrachtet wird. Würden die Nachhaltigkeitsregeln jedoch als Regeln im Sinne Alexys interpretiert, wäre dies mit der Anwendungspraxis des Konzepts konsistent. Dann könnten Defizite - als Differenz zwischen dem geforderten und dem vorliegenden Maß inhaltlicher Realisierung - in der Tat für einzelne Regeln separat bestimmt werden.

In dieselbe Richtung weisen die Beschreibungen der Nachhaltigkeitsregeln. Ein Beispiel: „Im Hinblick auf die erneuerbaren Ressourcen wird verlangt, dass deren Nutzungsrate ihre Regenerationsrate nicht übersteigen darf, wobei neben der Nutzungsintensität auch die Nutzungsart zu berücksichtigen ist (Regel 2.1)“ (Kopfmüller et al. 2001, S. 168). Unter welchen Bedingungen darf diese Regel als erfüllt gelten: wenn sie im höchstmöglichen Maße (Prinzip) oder wenn sie in einem bestimmten Maße (Regel) inhaltlich realisiert ist? Die Nachhaltigkeitsregel 2.1 trägt ein vergleichsweise hartes Kriterium ihres Erfüllt-Seins in sich: Ihrer Forderung ist genau dann Genüge getan, wenn die Nutzungsrate der erneuerbaren Ressourcen ihre Regenerationsrate nicht übersteigt. Dies spricht für eine Charakterisierung als Satisfizierungsnorm, d. h. als Regel. An anderer Stelle heißt es: „Nachhaltigkeit besteht substanziell in der dauerhaften Sicherung von Mindestbedingungen menschenwürdigen Lebens. Diese len Nachhaltigkeitsregeln sind nicht als Optimierungs-, sondern als Satisfizierungsgebote zu verstehen, oder, anders ausgedrückt, nicht als Prinzipien, sondern als Regeln im Sinne Alexys. Dieser Befund mutet ironisch an vor dem Hintergrund, dass das Konzept die Nachhaltigkeitsregeln unter Rekurs auf Alexy und in expliziter Abgrenzung zu dessen Regelbegriff als Prinzipien verstanden wissen will. Eine entsprechende Korrektur des Konzepts tut also not. Sie ist indes ,leicht zu haben“, denn das Konzept ist ja, wie oben ausgeführt, ohnehin vom Regelcharakter der Nachhaltigkeitsnormen durchtränkt. Lediglich zweierlei müsste geändert werden: zum ersten die explizite Charakterisierung der substanziellen Nachhaltigkeitsregeln als Prinzipien, zum zweiten die Ausführungen zum Umgang mit Zielkonflikten. Denn Zielkonflikte, d.h. Fälle divergierender Maßnahmenwirkungen, sind zwar nicht durch Abwägung zu lösen. Gleichwohl stellen sie ein zentrales Anwendungsproblem des Konzepts dar.

\section{Perspektiven: Szenarienbasierte Nachhaltigkeitsbewertung}

Ein zentrales Anwendungsproblem sind indes nicht nur Zielkonflikte, sondern Maßnahmenbewertungen generell: Es mangelt im Integrativen Konzept bislang an Hinweisen, unter welchen Bedingungen Maßnahmen insgesamt positiv oder negativ $\mathrm{zu}$ bewerten sind. Aufgrund der bereits angesprochenen Kluft zwischen dem Anspruchsniveau der Nachhaltigkeitsregeln und der Wirkmächtigkeit von Maßnahmen versteht sich dies nicht von selbst. Den normativen Horizont für Maßnahmenbewertungen stellt gewiss die Forderung dar, Mindestbedingungen menschenwürdigen Lebens $\mathrm{zu}$ schaffen und dauerhaft $\mathrm{zu}$ bewah-

\section{Die hier angeregte Korrektur des Integrativen Nachhaltigkeitskonzepts bietet große Chancen - nicht nur für seine theoretische Konsistenz, sondern gerade auch für seine Praktikabilität.}

Mindestbedingungen lassen sich unter drei ,generelle Ziele‘ der Nachhaltigkeit subsumieren und als substanzielle Nachhaltigkeitsregeln formulieren [...]“ (Kopfmüller et al. 2001, S. 118). Wenn die substanziellen Nachhaltigkeitsregeln „diese Mindestbedingungen" formulieren, die es dauerhaft zu sichern gilt, so können von der Formulierung der Nachhaltigkeitsregeln, wie sie in der Literatur zum Integrativen Konzept zu finden ist, keine Abstriche gerechtfertigt werden.

Daneben existieren noch einige weitere Hinweise, auf die an dieser Stelle aus Platzgründen nicht näher eingegangen werden kann. ${ }^{8}$ Das Ergebnis jedoch ist eindeutig: Die substanziel-

8 Für eine gründliche Analyse, auch der Unterscheidung von Kern- und Randbereichen der Nachhaltigkeitsregeln, siehe Dusseldorp (2017). ren. Fraglich ist, wie eine Brücke geschlagen werden kann zwischen dieser Forderung und einzelnen zur Bewertung stehenden Maßnahmen. Eine Antwort kann in normativen Szenarien einer nachhaltigen Entwicklung gefunden werden. Szenarienbasierte Nachhaltigkeitsbewertung zielt darauf, Maßnahmen in plausiblen Narrationen hin zur Realisierung von Mindestbedingungen menschenwürdigen Lebens einzubetten und darauf ihre Bewertung zu gründen. ${ }^{9}$ Sie trägt damit dem Umstand Rechnung, dass es bei Maßnahmenbewertungen nicht um Abwägung der Nachhaltigkeitsregeln geht, sondern vielmehr darum, ,das Ganze“ im Blick zu behalten.

9 Wie eine szenarienbasierte Nachhaltigkeitsbewertung konkret gestaltet werden könnte, wird in Dusseldorp (2017, S. 210-223) skizziert. 
Die hier angeregte Korrektur des Integrativen Nachhaltigkeitskonzepts bietet somit große Chancen - nicht nur für seine theoretische Konsistenz, sondern gerade auch für seine Praktikabilität. Maßnahmenbewertungen, der Anwendungsfall mit der größten Praxisrelevanz, würden möglich. Darüber hinaus könnte das Konzept mehr noch als jetzt für sich in Anspruch nehmen, sich tatsächlich für die Lösung derjenigen gravierenden Umwelt- und Entwicklungsprobleme zu engagieren, die seit rund drei Jahrzehnten unter dem Begriff einer nachhaltigen Entwicklung verhandelt werden. Dies ist nicht zuletzt auf einen funktionalen Unterschied zwischen Regeln und Prinzipien zurückzuführen, der in die Nachhaltigkeitsdebatte bislang keinen Eingang gefunden hat:

Nach March (1994) dienen Prinzipien dazu, aus einer Menge gegebener Handlungsalternativen die beste Alternative zu bestimmen - sie sind in diesem Sinne decision norms. Regeln hingegen fungieren als search norms: Sie leiten die Entwicklung von Handlungsalternativen an, die geeignet sind, den Norminhalt in hinreichendem Maße zu realisieren. Wenn die vorliegenden Handlungsalternativen nicht hinreichend sind, so ist dies als Ansporn zu verstehen, neue und besser geeignete Handlungsalternativen zu entwickeln. Dies ist es, worum es im Nachhaltigkeitskontext geht: Maßnahmen zu entwickeln, die in ihrer Gesamtheit hinreichend sind, um Mindestbedingungen menschenwürdigen Lebens zu realisieren.

\section{Literatur}

Alexy, Robert (1996): Theorie der Grundrechte. Frankfurt a. M.: Suhrkamp. Brown-Weiss, Edith (1989): In fairness to future generations. International law, common patrimony, and intergenerational equity. Tokyo: United Nations University.

Coenen, Reinhard; Grunwald, Armin (Hg.) (2003): Nachhaltigkeitsprobleme in Deutschland. Analyse und Lösungsstrategien. Berlin: edition sigma.
Dusseldorp, Marc (2017): Zielkonflikte der Nachhaltigkeit. Zur Methodologie wissenschaftlicher Nachhaltigkeitsbewertungen. Wiesbaden:

J. B. Metzler.

Jansen, Nils (1998): Die Struktur der Gerechtigkeit. Baden-Baden: Nomos. Kopfmüller, Jürgen (Hg.) (2006): Ein Konzept auf dem Prüfstand. Das integrative Nachhaltigkeitskonzept in der Forschungspraxis. Berlin: edition sigma.

Kopfmüller, Jürgen et al. (2001): Nachhaltige Entwicklung integrativ betrachtet. Konstitutive Elemente, Regeln, Indikatoren. Berlin: edition sigma.

March, James (1994): A primer on decision making. How decisions happen. New York: The Free Press.

Parodi, Oliver (2008): Technik am Fluss. Philosophische und kulturwissenschaftliche Betrachtungen zum Wasserbau als kulturelle Unternehmung. München: oekom.

Rösch, Christine; Bräutigam, Klaus-Rainer; Kopfmüller, Jürgen; Lichtner, Patrick; Stelzer, Volker (2016): Nachhaltigkeitsindikatoren zur Bewertung des deutschen Energiesystems. In: GAIA 25 (1), S. 69-70.

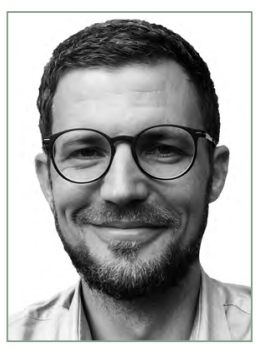

\section{DR. MARC DUSSELDORP}

ist wissenschaftlicher Mitarbeiter am Institut für Technikfolgenabschätzung und Systemanalyse (ITAS) des KIT. Studium der Geoökologie, Promotion in Philosophie. Forschungsschwerpunkte sind Technikfolgenabschätzung in den Bereichen Energie und Landwirtschaft sowie Facetten einer Theorie der TA. Lehrbeauftragter an verschiedenen Universitäten.

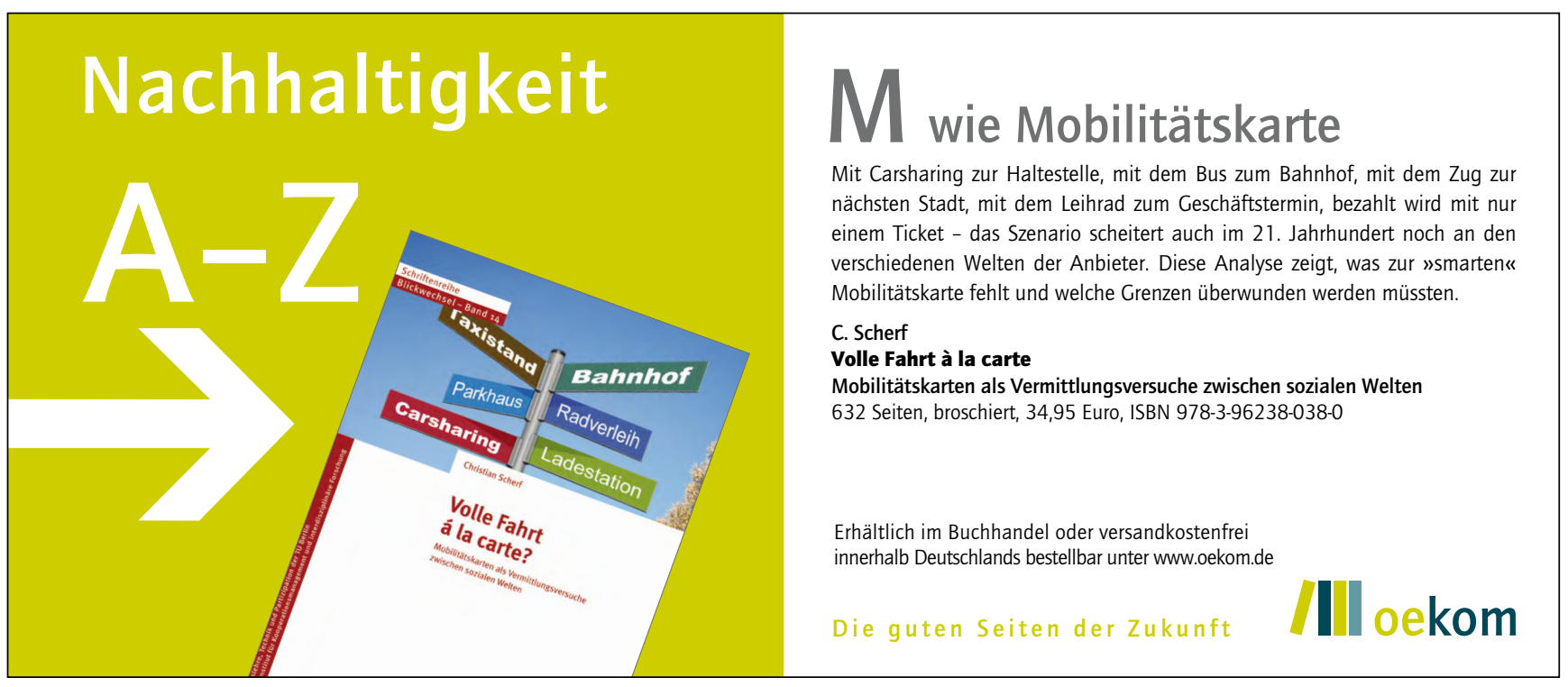

